\title{
Reality monitoring: Second perceptions and thoughts
}

\author{
MARCIA K. JOHNSON \\ State University of New York, Stony Brook, New York 11794 \\ CAROL L. RAYE \\ Barnard College, New York, New York 10027 \\ and \\ FRANCIS T. DURSO \\ State University of New York, Stony Brook, New York 11794
}

\begin{abstract}
Sentences were presented so that, for some, subjects heard the last word in the sentence and for others, they covertly filled in the last word. In addition, these critical externally presented or subject-generated words occurred either once or twice in the acquisition sequence. Later, subjects could better identify the origin of critical words that had occurred twice than the origin of those that had occurred once. Misidentifications of perceptions as thoughts were generally rare and unaffected by repetition. Repeating a thought reduced the likelihood that subjects would attribute it to external sources.
\end{abstract}

A classical idea (e.g., David Hume, William James) is that imagination and perception differ primarily in vividness or intensity. Along these lines, Johnson and Raye (in press, Note 1) have proposed that the memories derived from internally and externally generated events differ in that the class of externally derived memories typically have more contextual (time and place) information, more sensory information, and more meaningful detail represented in the memory compared with the class of memories for internally generated events. Johnson and Raye have also proposed that, in contrast, internally derived memories typically include more information about the cognitive operations that took place at the time the memory was established (e.g., imagery, search, decision processes, and so forth). These differences in typical class characteristics form the basis for a model of reality monitoring decisions, that is, decisions about the origins of memories. For example, a particular memory that is high in contextual information and low in information about cognitive operations should tend to be attributed to external sources.

It seems reasonable to suppose that practice or repetition increases "vividness," perhaps through the mechanism of making whatever contextual or sensory information is coded more accessible or by providing opportunities for more of such information to be stored. Thus, if repeating an internal generation resulted in a more vivid representation, it should be more likely to be misjudged as an external event. On the other hand, repetitions of an internal generation might be

This research was supported by a grant from the National Science Foundation. expected to strengthen those other attributes of the representation that could tend to identify it as internal (e.g., the codes for the operations involved in generating it). The following study attempted to determine which of these factors is more powerful, in the expectation that the results might point to the differential weighting given to various attributes in making the internalexternal decision.

A series of sentences was presented (e.g., "A chair is a piece of ____"), half with a pause that the subject covertly filled in and half presented with the pause filled (e.g., "A trout is a kind of fish"). In addition, half of the items of each type were repeated exactly within the study series (e.g., "A chair is a piece of "), and for the other half a new sentence with the same subject occurred in the series (e.g., "A trout is good to eat"). Thus the critical items (e.g., fish and furniture) occurred once or twice and the cue material (trout, chair) always occurred twice.

Subjects were then given a visual recognition list consisting of single words and were asked to classify each as new or old, and if old, as presented or generated. The question was whether, compared with items generated once, items that had been generated twice in the series would more often be mistakenly judged as presented. Since the second generation might be expected to be more effortless than the first, it might do little to increase cognitive operation information. If at the same time, repetition increased the "vividness" of the memory, repetitions should not increase the likelihood of later identifying the item as generated, and they might increase the likelihood of later misidentifying it as a presented item. (Two presentations of an external 
item as compared with one should not have a similar effect; that is, two presentations should not increase the chance that the origin of an external item is misjudged.) The opposite finding, namely, that generating the item twice increased the probability of correctly identifying its origin, would indicate that repetition increases information about cognitive operations in such a way as to outweigh potential increases in vividness in this situation.

\section{METHOD}

A number of sentences in which the context highly constrained the last word were created. Each of these was initially read to approximately 15 pilot subjects, who were asked to fill in the last word of each sentence. Any sentences for which there was not agreement among these subjects were discarded or modified; several new sentences were added to the list, and the same procedure was followed with a new set of 15 pilot subjects. Thus the sentences finally selected for the study were pretested to produce as much control as possible over what subjects would generate.

Ten items were randomly assigned to each of the four conditions described above: subject-generated (S) once, subjectgenerated twice, externally or experimenter-presented $(E)$ once, and experimenter-presented twice. These items were then ordered randomly with the restriction that the conditions be distributed evenly throughout the sequence. In order to equate materials, the items were rotated through the conditions, yielding four different acquisition lists. These lists were recorded on tape, with approximately $3 \mathrm{sec}$ between the end of one sentence and the beginning of the next. An initial four and final four sentences were added as buffer items and are of no further interest. An equal number of subjects were assigned to each list. During the first phase of the experiment, subjects were instructed to listen to each sentence, mentally filling in the last word when necessary, and, as a cover task to insure attention to each item, they were asked to rate each sentence for its "truth" value. The ratings were written on a sheet of paper, but otherwise subjects did not overtly respond to the items.

The test list consisted of the last word from each sentence ( 20 of which had actually been presented and 20 of which the subjects had generated themselves) and 20 new items selected to match the old items in frequency and concreteness. The items were randomly ordered, with the restriction that items from the four conditions and new items be evenly distributed throughout the test sequence. They were printed on a response sheet, preceded by several fillers. The subjects were paced through the recognition list at a 5 -sec rate and were instructed to indicate whether each item had been presented externally, had been generated by them, or was new. The interval between the end of the original list and the beginning of the test was approximately $2 \mathrm{~min}$. After the test, each subject was read all of the sentences with the last word omitted and was asked to fill in the last word. Intrusions were extremely rare (and in no case was there more than one per subject); therefore, it seems reasonable to conclude that subjects had thought the expected target items during acquisition.

\section{RESULTS AND DISCUSSION}

\section{Recognition}

Table 1 shows the mean number of misses (calling a word new when it was in fact old) for each of the four conditions. Repeating an item reduced the number of misses $[\mathrm{F}(1,23)=5.73, \mathrm{MSe}=1.86, \mathrm{p}<.025]$. Although there were slightly fewer misses for $\mathrm{S}$ items than for
Table 1

Mean Number of Misses

\begin{tabular}{lccc}
\hline & \multicolumn{3}{c}{ Number of Occurrences } \\
\cline { 2 - 4 } & 1 & 2 & Total \\
\hline Experimenter-Presented Words & 3.92 & 3.04 & 6.96 \\
Subject-Generated Words & 3.29 & 2.83 & 6.12 \\
Total & 7.21 & 5.87 & \\
\hline
\end{tabular}

E items, the main effect of source was not significant $[\mathrm{F}(1,23)=1.98, \mathrm{MSe}=2.10]$, nor was there an interaction between source and number of occurrences $[\mathrm{F}(1,23)<1]$. Thus $\mathrm{E}$ and $\mathrm{S}$ items profited approximately equally from a second occurrence with regard to being recognized as old.

\section{Identification of Origin}

With respect to false positives ( $E$ or $S$ responses to new items), the subjects were more likely to say a new item was externally presented $($ mean $=1.79)$ than to say they generated it (mean $=.83) \quad[\mathrm{F}(1,23)=9.22$, $\mathrm{MSe}=1.19, \mathrm{p}<.01]$.

The percent correct identification of origin, given a word was correctly recognized as old, was $72 \%$ for items occurring once and $79 \%$ for items occurring twice; repeating the item increased correct origin decisions $[\mathrm{F}(1,23)=6.36, \mathrm{MSe}=.01, \mathrm{p}<.02]$. Table 2 shows some further information that helps clarify the role of repetition in increasing origin identification in this task, the mean mistakes in identification of origin, given an item was recognized as old. A mistake for $\mathrm{E}$ items would be indicating they had been selfgenerated and a mistake for $\mathrm{S}$ items would be indicating they were $\mathrm{E}$ items. In this case, there were more $\mathrm{S}$ items called $\mathrm{E}$ items than the reverse $[\mathrm{F}(1,23)=24.44, \mathrm{MSe}=$ $1.69, \mathrm{p}<.001]$. This difference probably reflects the same tendency found with false positives: Familiar items with uncertain origin are attributed to an external source (see also Johnson, Raye, Foley, \& Foley, Note 2). More important, number of occurrences interacted with item type $[\mathrm{F}(1,23)=4.28, \mathrm{MSe}=1.07, \mathrm{p}<.05]$. As can be seen from Table 2 , this interaction reflects the fact that repetitions had little effect, if any, on the subjects' tendency mistakenly to say they had generated external items, but it reduced their tendency to attribute their thoughts to external sources. Thus in this context, repeating an $\mathrm{S}$ item apparently did not make it seem more like an externally presented item, but rather repetition increased the chances that the subject had available information that designated the item as internally generated. Since there was virtually no opportunity for idiosyncratically meaningful responses in these highly constrained contexts, the most reasonable explanation for the results is that origin decisions were based in part on information about cognitive operations, and repeating an operation increased its availability. This is consistent with the suggestion made by Raye, Johnson, 
Table 2

Mean Number of Origin Misidentifications

\begin{tabular}{lcc}
\hline & \multicolumn{2}{c}{ Number of Occurrences } \\
\cline { 2 - 3 } True Source & 1 & 2 \\
\hline Experimenter-Presented Words & .92 & 1.04 \\
Subject-Generated Words & 2.67 & 1.92 \\
\hline
\end{tabular}

and Taylor (1980) that one might expect cognitive operations information to weigh heavily in origin decisions about verbal materials.

While this seems the most likely interpretation, one possible alternative is that a second generation increases the availability of other information that can be used to correctly identify internal origin. For example, if the generated memory contained "sensory" information consisting of the item in the subject's own "voice," a decision might be based on the presence of this specific sensory information, and the availability of internalvoice information could be increased by repetition. To contrast these alternatives, subjects could be asked to think items in their own voice or in the experimenter's voice. If repetition of internally generated events increases identification of origin by increasing the availability of cognitive operations, performance should improve regardless of the voice of the generation. If the repetition helps by increasing the availability of sensory information specifically associated with thought, then thinking in the experimenter's voice should reduce or reverse the effects of repetition.

The outcome of this experiment provides an interesting contrast with some of our earlier studies (Johnson, Raye, Wang, \& Taylor, 1979; Johnson, Taylor, \& Raye, 1977; Raye et al., 1980). In those, a "rerepresentation" paradigm was used, in which subjects perceived items varying numbers of times and also generated the items varying numbers of times. The subjects were then asked to judge the presentation frequency of each item, ignoring the number of times they had generated it. The more times subjects generated an item, the higher their estimates of its presentation frequency. At first, the present finding that thinking a word twice increased correct identification of its origin may appear to contradict these earlier confusion functions. However, an important difference in the two procedures is that in the present study, the subject was regenerating an initially self-generated item, whereas in the earlier paradigm, the subject was always generating a rerepresentation of an initially externally presented event.

A reasonable working generalization might be that thinking repeatedly about things that have happened or happen repeatedly will produce inflated estimates of how often they actually happen. However, thinking repeatedly about something that never happens externally (e.g., a fantasy) may serve to increase the availability of the information that differentiates the generated from the perceived. Thinking of (generating in the sense of rerepresentation) an event that was formerly perceived is not like generating or thinking of an event that has not been specifically perceived. In both cases, the rerepresentation or regeneration should preserve or replicate characteristics of the original, and these characteristics should then be more available for origin decisions. For example, regenerating a previously generated event may make cognitive operations information more available, and (as in the present results) the subject should be more likely to decide later that this was a self-generated event. Thus, thinking about something once perceived should produce a trace more like a perceptually derived trace compared with thinking about something previously generated. This line of argument suggests that thinking and thinking of specific past events (external or internal) are quite different situations. Furthermore, the memorial consequences of "thinking of" depend not only on characteristics of processes operating at the time the new trace is established, but also on characteristics of previous processing.

\section{REFERENCE NOTES}

1. Johnson, M. K., \& Raye, C. L. A working model of reality monitoring. Paper presented at the annual meeting of the Psychonomic Society, November 1978.

2. Johnson, M. K., Raye, C. L., Foley, H., \& Foley, M. A. Cognitive operations and decision bias in reality monitoring. Manuscript submitted for publication, 1980.

\section{REFERENCES}

Johnson, M. K., \& RAYE, C. L. Reality monitoring. Psychological Review, in press.

Johnson, M. K., RAYE, C. L., WANG, A. Y., \& TAYloR, T. H. Fact and fantasy: The roles of accuracy and variability in confusing imaginations with perceptual experiences. Journal of Experimental Psychology: Human Learning and Memory, 1979, 5, 229-240.

Johnson, M. K., TAYLOR, T. H., \& RAYE, C. L. Fact and fantasy: The effects of internally generated events on the apparent frequency of externally generated events. Memory \& Cognition, $1977,5,116-122$.

RAYE, C. L., Johnson, M. K., \& TAYlor, T. H. Is there something special about memory for internally generated information? Memory \& Cognition, 1980, 8, 141-148.

(Received for publication April 23, 1980.) 